\title{
Les Oligochètes et Aphanoneura des eaux courantes de Tunisie. I - Données faunistiques
}

\author{
M. Boumaiza 1 \\ E. Martinez-Ansemil2 \\ ․ Giani $\mathbf{i}^{3}$
}

Mots clés: Oligochaeta, Aphanoneura, zoogéographie, Tunisie.

Les auteurs dressent une liste des Oligochètes et Aphanoneura récoltés en Tunisie. 34 genres ou espèces ont ainsi été récensés. Tous sont nouveaux pour la Tunisie et 7 especes sont nouvelles pour l'Afrique du Nord. Le peuplement est comparable à celui de la bordure nord de la Méditerranée mais il est plus pauvre.

Oligochaetes and Aphanoneura in streams of Tuntsia. I. Faunistic data.

Keywords : Oligochaeta, Aphanoneura, zoogeography, Tunisia.

The authors present a list of oligochaetes and Aphanoneura collected in Tunisia. 34 genera or species have now been recorded. All are new for Tunisia and 7 species are new records for North Africa. The population is similar to that of the northern coast of the Mediterranean but it is poorer.

Les Oligochètes aquatiques de Tunisie n'ont jusqu'à présent fait l'objet d'aucune étude particulière. Gauthier (1928) dans sa monographie sur les eaux continentales de l'Algérie et de la Tunisie signale 5 espèces d'Oligochètes pour l'Algérie mais aucune pour la Tunisie.

Dans son travail sur les eaux souterraines littorales et continentales de la Tunisie, Delamare Deboutteville (1953) a récolté de nombreux Oligochètes, mais aucun n'a été déterminé au niveau spécifique.

Au cours d'une étude sur les eaux courantes de la Tunisie, réalisée par l'un des auteurs (M. Boumaiza) 9289 Oligochètes ont été récoltés. L'étude de ce materiel nous permet de présenter ici un premier inventaire du peuplement en Oligochètes aquatiques de la Tunisie.

1. Laboratoire d'Ecologie et de Biologie Animale, Département des Sciences Biologiques, Faculté des Sciences de Tunis, Tunisie.

2. Colegio Universitario de Orense, C. General Franco, Orense, Espagne.

3. Laboratoire d'Hydrobiologie, UA 695 C.N.R.S., Université P. Sabatier, 118, route de Narbonne, 31062 Toulouse Cedex, France.

\section{Méthodes}

Les récoltes ont été effectuées entre 1980 et 1984. Elles concernent 49 stations réparties sur la plus grande partie du réseau hydrographique tunisien. de l'extrême nord jusqu'à la limite méridionale du Chott El Djérid. Ces récoltes ont été effectuées exclusivement sur des milieux en eaux courantes.

Les prélèvements benthiques ont été réalisés à l'aide d'un filet de Surber de $300 \mu$ m de vide de maille. Ils ont été complétés par des récoltes effectuées à l'aide de filets pour la dérive de 200 à 300 $\mu \mathrm{m}$ de vide de maille.

Au cours de la période d'étude, 105 prélèvements ont été effectués. Ils se répartissent ainsi dans le temps :

-68 prélèvements au printemps (mars, avril, mai) : 1829 individus récoltés;

-8 en été (juin, juillet) : 888 individus ;

- 11 en automne (novembre) : 322 individus ;

-18 en hiver (décembre, janvier, février) : 2044 individus. 


\section{Les stations de récoltes}

Ces stations ont été décrites de façon détaillée dans un travail précédent (Boumaiza 1984). Aussi nous dresserons ici une liste de ces stations regrou. pées par bassin versant et nous préciserons uniquement l'altitude (A) et la conductivité électrique de l'eau (C) mesurée en micro Siemens $/ \mathrm{cm}$ à $20^{\circ} \mathrm{C}$ pour chacune de ces localités. Le lecteur intéressé trouvera dans le travail précédemment cité les données complémentaires concernant la description de ces stations dont la localisation est précisée sur la fig. 1.

\subsection{Stations de Tunisie septentrionale}

\subsubsection{Bassin de la Medjerda}

- Station 1 : Oued Medjerda à proximité de la ville de Jendouba $(A=146 \mathrm{~m}, C=1127-2371)$.

- Station 2: Oued Medjerda à El Bathan $(A=28 \mathrm{~m}$, $\mathrm{C}=1515-4001$ ).

- Station 3: Oued Mellègue (affluent de la Medjerda) à environ $18 \mathrm{~km}$ au sud-est du village de Sakiet Sidi Yous. sef $(A=329 \mathrm{~m}, \mathrm{C}=6132-6350)$.

- Station 4 : Oued Mellègue à environ $12 \mathrm{~km}$ au sud de la ville de Jendouba, sur la route de Jendouba à El Kef. (A $=169 \mathrm{~m}, \mathrm{C}=2600-3774)$.

- Station 5 : Oued Mellègue à 1 ou $2 \mathrm{~km}$ du confluent avec la Medjerda et à 6 ou $7 \mathrm{~km}$ au nord-est de la ville de Jendouba $(A=138 \mathrm{~m}, C=2356-4158)$.

- Station 6: Oued Tessa (afluent de la Medjerda) à environ $18 \mathrm{~km}$ au sud-ouest du Krib $(\mathrm{A}=295 \mathrm{~m}, \mathrm{C}=$ 1550-4444).

- Station 7 : Oued Ghézala (affluent secondaire de la Medjerda) à environ $2 \mathrm{~km}$ au sud de la localité de Fernana $(\mathrm{A}=230 \mathrm{~m}, \mathrm{C}=398 \cdot 1024)$.

- Station 8: Oued Bouheurtma (affluent de la Medjerda) à $3 \mathrm{~km}$ à l'ouest de la ville de Bou Salem et à 500 m du confluent avec la Medjerda $(A=133 \mathrm{~m}, \mathrm{C}=1266)$.

- Station 9 : Oued Kesseb (affluent de la Medjerda) à environ $3 \mathrm{~km}$ à l'est de la ville de Bou Salem et à $3-4 \mathrm{~km}$ du confluent avec la Medjerda $(A=134 \mathrm{~m}, C=517-1061)$,

- Station 10 : Oued Béja (affluent de la Medjerda) à 3-3,5 $\mathrm{km}$ au nord-est de la ville de Béja, sur la route de Béja à Mateur ( $A=155 \mathrm{~m}, C=730$ ).

- Station 11 : Oued béja à 3-3,5 km à l'est de Béja et à $15,5 \mathrm{~km}$ du confluent avec la Medjerda $(A=144 \mathrm{~m}, \mathrm{C}$ $=846-(177)$. Cette station est caractérisée par une forte pollution due aux rejets urbains et surtout aux rejets d'une sucrerie située en amont.

- Station 12: Oued Zargua (affluent de la Medjerda) à $5 \mathrm{~km}$ au nord-ouest de la localité du même nom et à $4 \mathrm{~km}$ de la confluence avec la Medjerda $(A=95 \mathrm{~m}, \mathrm{C}=$ 1467-3217).

- Station 13 : Oued Khalled (affluent de la Medjerda) à environ $7 \mathrm{~km}$ au nord-est de la ville de Tébousouk $(A=$ $230 \mathrm{~m}, \mathrm{C}=2099-3571$ ).
- Station 14 : Oued Siliana, sur le cours supérieur, à environ $2 \mathrm{~km}$ à l'est de la ville de Siliana $(A=420 \mathrm{~m}, \mathrm{C}$ $=1277-1628$ ).

- Station 15 : Oued Siliana à $12-13 \mathrm{~km}$ au nord-ouest de Siliana et à $10-11 \mathrm{~km}$ au sud de Lakhouat $(A=375 \mathrm{~m}$. $\mathrm{C}=1949$ ).

- Station 16 : Oued Siliana à proximité de la ville de Gaafour ( $A=239 \mathrm{~m}, \mathrm{C}=2262$ ).

- Station 17: Oued Siliana à $1 \mathrm{~km}$ à l'ouest d'El Arroussa $(A=175 \mathrm{~m} \cdot \mathrm{C}=7498)$.

- Station 18 : Oued Siliana à 2-2,5 $\mathrm{km}$ au sud-ouest de Testour et à $1 \mathrm{~km}$ environ du confluent avec la Medjerda $(A=78 \mathrm{~m}, \mathrm{C}=6504-6.895)$.

- Station 19: Oued Ramla (affluent de l'Oued Siliana) sur la route d'Ousslatia à environ $8 \mathrm{~km}$ au sud de la ville de Siliana $(A=470 \mathrm{~m}, \mathrm{C}=962)$.

\subsubsection{Bassins versants du Nord-ouest de la Tunisie}

- Station 20: Oued Ennour (affluent secondaire de l'Oued El Kébir) à $2 \mathrm{~km}$ environ de Babouch sur la route de la station thermale d'Hamman Bourguiba $(A=400 \mathrm{~m}$, $\mathrm{C}=$ 249-449).

- Station 21: Oued Barbar à proximité de la station thermale d'Hamman Bourguiba ( $A=400 \mathrm{~m}, C=1175$ ).

- Station 22 : Oued El Kébir à $45 \mathrm{~km}$ au sud de la ville de Tabarka et à $5 \mathrm{~km}$ enviton de l'embouchure $(A=8 \mathrm{~m}$, $\mathrm{C}=45(-714)$.

- Station 23: Oued Renagha (affluent de l'oued E] Kébir) à $300-400 \mathrm{~m}$ en amont du confluent $(A=65 \mathrm{~m}, \mathrm{C}$ $=760-1100$ ).

- Station 24 : Oued Bouterfes (cours d'eau temporaire à l'est de Tabarka) à $3-4 \mathrm{~km}$ de l'embouchure, sur la route de Nefza à Tabarka ( $A=23 \mathrm{~m}, \mathrm{C}=1082)$.

- Station 25: Oued Melah (Ouchtata), affluent d'Oued Zouara, à Ouchtata à $8 \mathrm{~km}$ de sa confluence $(A=29 \mathrm{~m}$, $\mathrm{C}=1792 \mathrm{~m})$.

- Station 26: Oued El Madène (affluent de l'Oued Zouara) à une dizaine de $\mathrm{km}$ de la confluence avec l'Oued Zouara et à proximité de Nefza $(\mathbf{A}=30 \mathrm{~m}, \mathbf{C}=717)$.

- Station 27 : Oued Ziatine à l'est de Cap Ferrat et à $3 \mathrm{~km}$ de l'embouchure $(A=6 \mathrm{~m}, \mathrm{C}=309-722)$.

\subsubsection{Bassin versant du lac Ichkeul}

- Station 28 : Oued Sedjenane, sur la route du Cap Ser. rat, à une trentaine de kilomètres de l'embouchure $(A=$ $100 \mathrm{~m}, \mathrm{C}=445-3192$ ).

- Station 29 : Oued Melah à environ $8 \mathrm{~km}$ au nord-ouest de Djalta $(A=15 \mathrm{~m}, \mathrm{C}=760-8142)$

- Station 30 : Oued Melah (Teskraya) sur la route de Teskraya à $8 \mathrm{~km}$ environ de l'embouchure $(\mathrm{A}=10 \mathrm{~m}, \mathrm{C}$ = 3895).

- Station 31 : Oued Ghézala, petit cours d'eau temporaire au sud-ouest du lac Ichkeul, à $5 \mathrm{~km}$ de l'embouchure ( $A=22 \mathrm{~m}, \mathrm{C}=992$ ).

- Station 32 : Oued Joumine, à $1 \mathrm{~km}$ environ de la vil le de Mateur $(A=15 \mathrm{~m}, C=524-1525)$.

- Station 33 : Oued Tine (affluent de l'Oued Joumine) à $9 \mathrm{~km}$ environ au sud-est de Mateur $(A=39 \mathrm{~m}, \mathrm{C}=$ 1994-4238). 


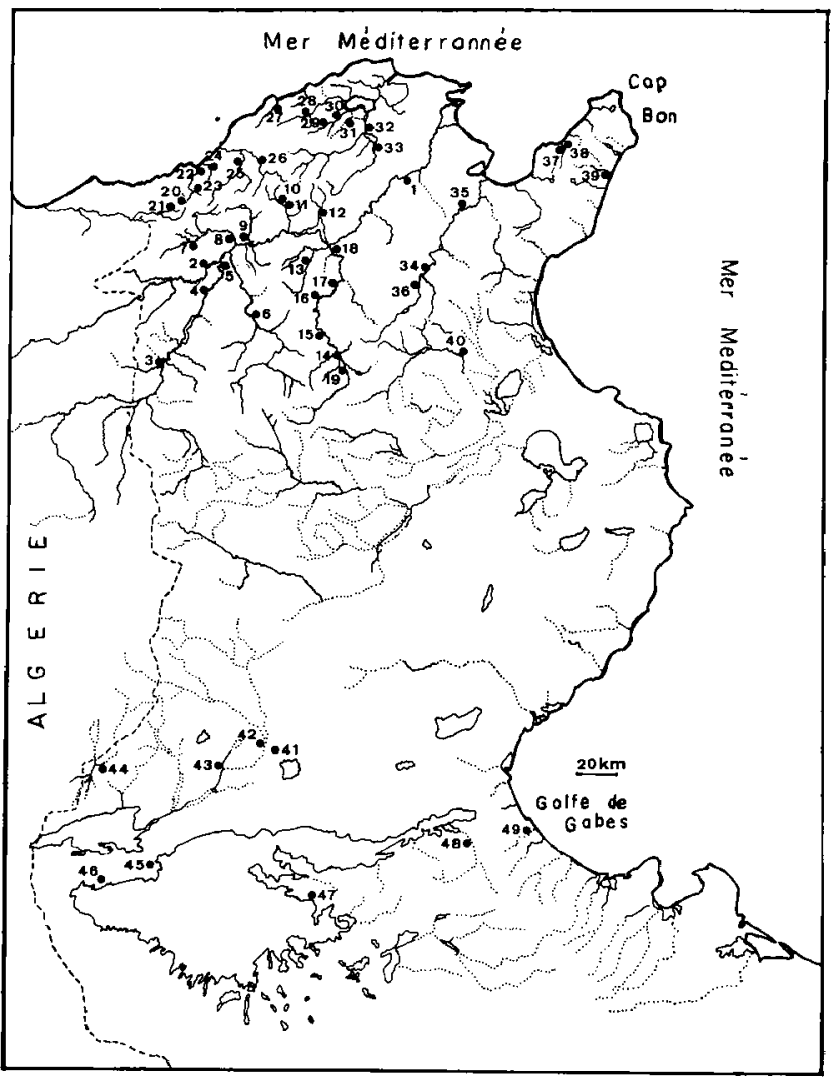

Fig. 1. Localisation des stations d'étude.

\subsubsection{Cours d'eau du nord-est de la Tunisie}

- Station 34 : Oued Miliane, sur le cours supérieur à $2-2,5 \mathrm{~km}$ a nord d'El Fahs $(\mathrm{A}=168 \mathrm{~m}, \mathrm{C}=2629-4058)$.

- Station 35 : Oued Miliane, sur le cours inférieur à 4-5 $\mathrm{km}$ 'au sud-est de La Mohammadia $(\mathrm{A}=34 \mathrm{~m}, \mathrm{C}=$ 2046-6844).
- Station 36 : Oued El Kébir (affluent de l'Oued Miliane) à environ $3 \mathrm{~km}$ au sud de la ville d'El Fahs $\left(A=192 \mathrm{~m}_{1}\right.$ $\mathrm{C}=1070-1991$ ).

- Station 37: Oued El Abid, sur le cours inférieur à environ $3 \mathrm{~km}$ de l'embouchure, en amont d'un pont $(\mathbf{A}=$ $12 \mathrm{~m}, \mathrm{C}=922-2563$ ). 
- Station 38 : station contigue à la précédente mais en aval du pont ( $\mathrm{A}=12 \mathrm{~m}, \mathrm{C}=2835-15623$ ).

- Station 39 : Oued Lebna à $1.2 \mathrm{~km}$ au sud-ouest du village de Menzel Heurr $(A=7 \mathrm{~m}, \mathrm{C}=$ 1423-11907).

\subsection{Stations de Tunisie centrale}

- Station 40: Oued Nebhana à $9-10 \mathrm{~km}$ au nord du village de Sbikha $(A=92 \mathrm{~m}, \mathrm{C}=2686-2720)$.

- Station 41 : Oasis Lella (Aîn Soltane) à $6 \mathrm{~km}$ environ au sud-est de la ville de $\mathrm{Gaf} s a(\mathrm{~A}=275 \mathrm{~m}, \mathrm{C}=1574-1888)$.

- Station 42 : Oasis Gafsa à environ une centaine de metres de la source, à Gafsa $(A=290 \mathrm{~m}, \mathrm{C}=1923)$.

\subsection{Stations de Tunisie méridionale}

- Station 43: Oued Magroune ( $=0$. Melah) sur la route de Gafsa à Metlaoui, à $21-22 \mathrm{~km}$ au sud-ouest de la ville de Gafsa $(A=159 \mathrm{~m}, \mathrm{C}=31715.36188)$.

- Station 44: Oued Tamerza sur la route Tozeur. Tamerza à 1 ou $2 \mathrm{~km}$ au sud de la ville de Tamerza $(\mathrm{A}=$ $3(00 \mathrm{~m}, \mathrm{C}=3295)$.

- Station 45 : Oasis Tozeur à Tozeur, au nord-ouest du Chot El Djérid (A $=50 \mathrm{~m}, \mathrm{C}=2743-3139$ ).

- Station 46 : Oasis Nefta, sur le cours de l'Oued Kentara, à Nefta $(A=75 \mathrm{~m}, \mathrm{C}=3572-4200)$.

- Station 47 : Oasis Kébili (Ras El Aïn) sur le cours de l'Aîn Chems à Kébili, à quelques dizaines de mètres d'un puits artésien $(A=41 \mathrm{~m}, \mathrm{C}=2750-2885)$.

- Station 48 : Oued El Hamma, sur la route de Gabès à El Hamma à proximité de la localité de Sombat $(A=52$ $\mathrm{m}, \mathrm{C}=4444-4692$ ).

- Station 49 : Oasis Chénini à $45 \mathrm{~km}$ au sud-ouest de la ville de Gabès, à proximité de Chénini $(\mathrm{A}=23 \mathrm{~m}, \mathrm{C}=$ 3564-3674)

\section{Inventaire faunistique}

Pour chacune des espèces recensées nous préciserons le nombre d'individus examinés (n) et les stations où elles ont été récoltées.

\subsection{Oligochaeta}

\subsubsection{Haplotaxidae}

Haplotaxis gordioides (Hartmann, 1821) $\mathrm{n}=3$ stations : 12, 29.
Afrique du Nord: Algérie, Maroc (Gagneur \& al 1986, Martinez-Ansemil \& Giani 1985).

\subsubsection{Lumbriculidae}

Trichodrilus allobrogum Claparède, $1862 n=41$. Stations : 10, 29.

Afrique du Nord : Algérie, Maroc (Gagneur \& al 1986, Martinez-Ansemil \& Giani 1985).

\subsubsection{Naididae}

Chaetogaster diastrophus (Gruithuisen, 1828) $n=1$. Station : 37.

Afrique du Nord : Algérie (Maupas cité par Seurat 1930, Gagneur \& al 1986).

Paranais birsteini Sokolskaya, 1971. $\mathrm{n}=2666$ Stations : $2,3,4,6,8,12,13,14,15,16,17,18,19$, $25,29,30,31,33,34,35,37,39,40,41,44,48$.

Afrique du Nord: Algérie, Maroc (Gagneur \& al 1986. Martinez-Ansemil \& Giani 1985).

Cette forme a été récoltée en assez grande abondance dans toute l'Afrique du Nord (Maroc, Algérie, Tunisie) et elle a été considérée comme une variété - var. magrhebensis - de Paranais birsteini par Martinez-Ansemil \& Giani (1985).

Nais communis Piguet, $1906 \mathrm{n}=78$.

Stations: 7, 13, 14, 20, 21, 22, 27, 37, 39, 41, 44 .

Afrique du Nord : Algérie (Gagneur \& al 1986).

Nais elinguis Müller, $1773 \mathrm{n}=1534$.

Stations: $1,2,6,7,8,10,12,13,14,15,18,19,21$. $22,23,26,28,30,34,35,38,41,44,47,48$.

Afrique du Nord: Algérie, Maroc (Gauthier 1928, Maupas cité par Seurat 1930, Gagneur \& al 1986; Martinez-Ansemil \& Giani 1985).

Nais pardalis Piguet, $1906 \mathrm{n}=789$

Stations: $1,7,14,20,21,27,32,34$.

Afrique du Nord : Algérie, Maroc, (Gauthier 1928, Gagneur \& al 1986, Martinez-Ansemil \& Giani 1985).

Nais variabilis Piguet, $1906 \mathrm{n}=63$

Stations : 2, 10, 12, 20, 37, 39, 47.

Afrique du Nord : Algérie (Gauthier 1928, Gagneur $\&$ al 1986).

Ophidonais serpentina (Müller, 1773) $n=5$

Station : 27.

Cette espèce est nouvelle pour l'Afrique du Nord. 
Dero obtusa d'Udekem, $1855 \mathrm{n}=91$

Stations : 10, 37, 42.

Cette espèce est nouvelle pour l'Afrique du Nord.

Pristina aequiseta f. aequiseta Bourne, $1891 \mathrm{n}=5$ Stations : $7,37$.

Afrique du Nord : Algérie (Maupas cité par Seurat 1930, Gagneur \& al 1986).

Pristina aequiseta Bourne, 1891 f. foreli (Piguet, 1906) $\mathrm{n}=\mathbf{5}$

Stations : 20, 21.

Afrique du Nord : Algérie (Gagneur \& al 1986).

Pristina longiseta Ehrenberg, I828 $n=2$

Stations : 20, 37.

Afrique du Nord : Algérie (Gagneur \& al 1986).

Pristina menoni (Aiyer, 1929) $\mathrm{n}=1346$

Stations : 2, 3, 4, 6. 7. 10, 12, 13, 14, 15. 16, 18, 19 , $20,21,23,24,25,28,29,31,32,35,36,37,40,41$, $44,46$.

Afrique du Nord : Algérie, Maroc (Gagneur \& al 1986; Martinez-Ansemil \& Giani 1985).

Pristina sima (Marcus, 1944) $\mathbf{n}=1$

Station : 20.

Afrique du Nord : Algérie (Gagneur \& al, 1986).

\subsubsection{Tubificidae}

Tubifex ignotus (Stolc, 1886) $\mathbf{n}=310$

Station : 43.

Cette espèce est nouvelle pour l'Afrique du Nord.

Tubifex tubifex (Müller, 1774) $n=1792$

Nous avons récolté en Tunisie les 4 formes de cette espèce.

Inbifex tubifex f. Iubifex $\mathrm{n}=926$

Stations : $2,8,10,11,13,14,17,25,34,37,40,41$. 47, 48.

Afrique du Nord : Algérie, Maroc, (Gauthier 1928. (iagneur \& al 1986, Martinez-Ansemil \& Giani 1985).

7. abifex f. blanchardi $\mathbf{n}=461$

Stations: 2, 11, 15, 31, 41, 44, 45, 47, 49.

Afrique du Nord : Algérie, Maroc (Vejdovsky 1891, Gauthier 1928, Gagneur \& al 1986, Martinez-Ansemil \& Giani 1985).

T. tubifex $f$. bergi $\mathrm{n}=\mathbf{4 0 4}$

Stations: 10, 11, 13, 31, 41 .

Afrique du Nord: Algérie, Maroc (Gagneur \& al 1986, Martinez-Ansemil \& Giani 1985).
$T$. tubifex $f$. grandiseta $\mathrm{n}=1$

Station : 20.

Afrique du Nord: Algérie, Maroc (Gagneur \& al 1986, Martinez-Ansemil \& Giani 1985).

La présence des formes bergi et blanchardi est liée à la forte minéralisation des eaux de certains des cours d'eau prospectés ( $\mathrm{cf}$. $\$ 4$ ). La forme grandiseta décrite par Rodriguez. (1984) d'Espagne a maintenant été observée dans toute l'Afrique du Nord.

Limnodrilus hoffmeisteri Claparède, $1862 \mathrm{n}=25$ Stations : 8, 9, 15, 31.

Afrique du Nord: Algérie, Maroc (Gagneur \& al 1986, Martinez-Ansemil \& Giani 1985).

Limnodrilus udekemianus Claparède, $1862 \mathrm{n}=25$

Stations : 5, 10, 11, 33, 34, 41.

Afrique du Nord: Algérie, Maroc (Gagneur \& al 1986, Martinez-Ansemil \& Giani 1985).

Potamothrix bavaricus (Oschmann, 1913) $n=50$

Stations: 34, 35, 37, 39.

Afrique du Nord: Algérie, Maroc (Gagneur \& al 1986, Martinez-Ansemil \& Giani 1985).

Psammoryctides barbatus (Grube, 1861) $\mathbf{n}=\mathbf{2}$

Stations:

Afrique du Nord : Algérie, (Gagneur \& al 1986).

Aulodrilus pigueti Kowalewski, $1914 \mathrm{n}=44$

Stations : 10, 31, 37.

Cette espèce est nouvelle pour l'Afrique du Nord.

Aulodrilus pluriseta (Piguet, 1906) $\mathrm{n}=3$

Station : 10.

Afrique du Nord: Algérie, Maroc (Gagneur \& al 1986, Martinez-Ansemil \& Giani 1985).

Epirodrilus moubayedi Giani \& Martinez-Ansemil, $1983 \mathrm{n}=5$

Stations : 10, 29, 37.

Cette espèce est nouvelle pour l'Afrique du Nord. Elle n'était préalablement connue que du Liban et elle était considérée comme caractéristique du Levant (Giani \& Martinez-Ansemil 1983, MartinezAnsemil \& Giani 1985). Sa découverte en Tunisie est donc intéressante et semble suggérer que cette espèce est peut-être largement distribuée sur le pourtour de la Méditerranée.

\subsubsection{Enchytraeidae}

La grande difficulté lièe à l'étude d'animaux fixés pour la détermination des repésentants de cette farnille ne nous a pas permis d'identifier au niveau spécifique une grande partie du matériel récolté. 
Buchholzia fallax Michaelsen, $1887 \mathrm{n}=2$

Stations : 10, 32.

Cettc espèce cst nouvelle pour l'Afrique du Nord.

Buchholzia sp. $\mathrm{n}=2$

Stations: 19, 26.

Ces spécimens ne peuvent être attribués à l'espèce B. africana décrite d'Algérie par Cernosvitov (1933). Ils appartiennent probablement à la même espèce que ceux que nous avons observés en Algérie (Gagneur \& al 1986).

Enchytraeus buchholzi Michaelsen, $1887 \mathrm{n}=11$

Stations: 7, 12, 13, 14, 22, 31.

Cette espèce est nouvelle pour l'Afrique du Nord.

Henlea sp. $\mathrm{n}=1$

Station : 13.

Fridericia spp. $\mathrm{n}=6$

Stations: 10, 13, 20, 31, 40.

Marionina riparia Bretscher, 1899 augm. Cernosvitov, $1928 \mathrm{n}=4$

Stations: 22, 23, 39.

Afrique du Nord: Algérie, Maroc (Gagneur \& al 1986, Martinez-Ansemil \& Giani 1985).

Marionina spp. $\mathrm{n}=102$

Stations: 10, 12, 13, 14, 18, 21, 31, 32, 40, 41.

\subsubsection{Criodrilidae}

Criodrilus lacuum Hoffmeister, $1845 n=15$

Station: 41.

A notre connaissance cette espèce est nouvelle pour l'Afrique du Nord.

\subsection{Aphanoneura}

\section{Aeolosomatidae}

Aeolosoma travancorense Aiyer, $1926 \mathrm{n}=1$

Station : 7.

A notre connaissance cette espèce est nouvelle pour l'Afrique du Nord.

Aeolosoma sp. $\mathrm{n}=1$

Station : 27.

\section{Conclusions}

Sur les 9289 spécimens récoltés, seulement 256 individus immatures (essentiellement des Tubificidae) n'ont pas été examinés. 9030 Oligochètes au total ont donc été étudiés. Ils se répartissent ainsi :
- 6586 Naididae soit $72,9 \%$ des Oligochètes.

- 2256 Tubificidae $(24,9 \%)$.

- 130 Enchytraeidae $(1,4 \%)$.

- $\quad 41$ Lumbriculidae $(0,45 \%)$.

- $\quad 15$ Criodrilidae $(0,16 \%)$.

- 3 Haplotaxidae.

Les Naididae et les Tubificidae représentent à eux seuls $97,9 \%$ des récoltes. Mais notons que cinq espèces appartenant à ces deux familles forment à elles seules $90 \%$ du nombre total d'individus récoltés. Ce sont, par ordre d'importance décroissante $P a$. birsteini, T. tubifex, N. elinguis, Pr. menoni et $N$. pardalis. Les 4 dernières formes sont très ibiquistes. Au contraire, $P a$. birsteini, qui représente à elle seule près du quart des Oligochètes récoltés $(22,8 \%)$ n'était jusqu'à présent connue que du littoral marin. Sa présence et son abondance dans les eaux continentales de la Tunisie et de toute l'Afrique du Nord esı donc à souligner. Elle est à mettre eñ relation avec la forte conductivité des eaux, voire les fortes chlorosités, observées dans les cours d'eau de cette région du globe. Ceci est corroboré par l'abondance de $N$. elinguis bien connue pour sa préférence pour les eaux fortement minéralisées ou saumâtres ainsi que par la présence des formes bergi et blanchardi de T. tubifex. En effet Chapman \& Brinkhurst (1985) ont montré expérimentalement que l'augmentation de la dureté de l'eau, où des valeurs extrêmes du $\mathrm{pH}$ entrainaient la disparition progressive des soies capillaires et des denticulations intermédiaires des crochets et que les individus se transformaient progressivement pour donner la forme bergi puis la forme blanchardi.

L'abondance de Pr. menoni est aussi probablement liée aux fortes minéralisations de l'eau car nous avons déjà observé cette espèce dans des milieux fortement pollués (Giani 1984).

Ce premier inventaire fait état de $\mathbf{3 6}$ taxons qui sont tous nouveaux pour la faune de Tunisie qui était totalement inconnue. 7 espèces d'Oligochètes et $1 \mathrm{~d}$ 'Aphanoneura sont nouvelles pour la faune d'Afrique du Nord. Il s'agit d'O. serpentina, $T$, ignotus, A. pigueti, E. moubayedi, B. fallax, E. buchholi, $C$. laccum et $A$. travancorense Cette étude porte donc à 54 le nombre d'espèces recensées en Afrique du Nord. L'inventaire des Oligochètes de Tunisie est donc loin d'être exhaustif.

Les Naididae sont largement dominants et avec 12 especes appartenant à 6 genres ils constituent la 
famille la plus diversifiée. Les genres Nais et Prisina qui regroupent 8 espèces à eux deux sont les mieux représentés comme c'était également le cas en Algérie (Gagneur \& al 1986). A l'exception de Pa. birsteini et Pr. sima toutes les formes de Naididae récoltées sont des espèces cosmopolites ou à large répartition. Seule la présence de Pa. birsteini est intéressante à souligner. Cette espèce est présente dans toute l'Afrique du Nord alors qu'elle n'était précédemment connue que de la bordure du Pacifique (Brinkhurst \& Coates 1985).

Les Tubificidae constituent le $2^{c}$ groupe en abondance mais également du point de vue de la diversité : 6 genres et 9 espèces. Il s'agit essentiellement de formes à très large répartition mondiale. Seule la présence d'E. moubayedi est à souligner: cette espèce n'était en effet connue que du Liban.

Les Enchytraeidae ne représentent que $1,4 \%$ de l'effectif global mais ils sont cependant relativement bien diversifiés : 5 genres et au moins 8 espèces recensées.

Les autres familles (Lumbriculidae, Criodrilidae, Haplotaxidae et Aeolosomatidae) représentent ensemble moins de $1 \%$ du peuplement. Le seul point à noter est la faible représentation des Lumbriculidae comme nous l'avions d'ailleurs observee dans le's autres pays d'Afrique du Nord (Gagneur et al 1986, Martinez-Ansemil \& Giani 1985).

Le peuplement des Oligochètes de la Tunisie, bien que certainement incomplètement connu, est relativement pauvre. Il est composé en majorité d'espè. ces à très large répartition. Il ne comporte aucun élément d'origine Ethiopienne et il est très semblable à celui de la bordure nord de la Méditerranée mais en plus pauvre. La différence essentielle entre ces deux peuplements réside dans la faible diversité des Lumbriculidae en Afrique du Nord. Ces observations sur le peuplement tunisien corroborent celles précédemment effectuées lors de l'étude des Oligochètes d'Algérie (Gagneur \& al 1986).

Travaux cliés

Brinkhurst (R.O.) \& Coates (K.) 1985. - The genus Paranais (Oligochaeta : Naididae) in North America. Proc. Biol. Soc. Wash. $98(2): 303-313$

Cemosvitov (L.). 1933. - Note sur les Oligochètes d'Algérie. Bull. Soc. Hist. Nat. Afriq. Nord., 24 : 255-258.

Chapman (P.M.) \& Brinkhurst (R.O.). 1985. - Hair today. gone tomorrow (Induced setal changes in tubificid Oligochaetes) 3-rd Intemational Symposium on Aquatic Oligochaeta, Ham. burg, sep. 29 . oct. 4, 1985 (Abstracts).

Deiamare-Deboutteville (C.), 1953. - La faune des eaux souterraines littorales des plages de Tunisie. Vie et milieu, 4 (2) : 141-170.

Gagneur (J.), Giani (N.) \& Martinez-Ansemil (E.). 1986. - Les Oligochètes aquatiques d'Algérie. Bull. Soc. Hist. nat. Toulouse 122: $119-124$

Gauthier (H.). 1928. - Recherches sur la faune des eaux continenlales de l'Algéne el de la Tumisie. Alger, imprimerie Minerva, 149 p. 3 pl. hors-texte.

Giani (N.). 1984, - Le Riou Mort, affluent du Lot, pollué par métaux lourds. IV. Etude des Oligochètes. Annls Limnol., 20 (3) : 167.181 .

Giani (N.) \& Martinez-Ansemil (E.). 1983. - Les Oligochetes aquatiques du Liban. IV. Epirodriltis moubayedi $\mathrm{n}$. sp. (Tubificidae Rhyacodrilinae). Annls Limmol, 19 (2) : 87-92.

Martinez-Ansemil (E.) \& Giani (N.) 1985. - The distribution of aquatic Oligochaetes in the South and eastem mediterranean area. $3 \cdot$ rd International Symposium ont Aquatic Oligochaeta. Hamburg. Sept. 29 - Oct. 4, 1985 (sous presse)

Rodriguez (P.). 1984. - Estudio taxonomico de los Oligoquetos acuaticos del Pais Vasco y cuenca alta del Ebro y caracteriza tion de las Communidades de los rios Nenion y Butron (Viscaya). Tesis doctoral, Université de Bilbao, 348 p.

Seurat (L.G.) - Oligochètes limicoles, in Exploration zoologique de l'Algerie de 1830 a 1930. Collection du centenaire de l'Algérie. Masson éd. Paris : 570.571.

Vejdovsky (F.). 1981. Note sur un Tubifex d'Algérie. Mém. Soc. Zool. Fr., $4: 596-603$. 